\title{
Carrier Dynamics Probed by Noise in High-Frequency Electronic Devices
}

\author{
Tomás González \\ Departamento de Física Aplicada \\ Universidad de Salamanca \\ Salamanca, Spain \\ e-mail: tomasg@usal.es
}

\begin{abstract}
Electronic noise, despite being a limiting factor in many applications of semiconductor devices and integrated circuits, constitutes also a source of information about the microscopic processes taking place inside the devices. The level of noise and its frequency dependence can provide very useful knowledge about the dynamics of charge carriers, not available from the DC behavior. Monte Carlo simulations, performed precisely at a microscopic level, can be used to identify the link between the noise behavior of the devices and the peculiarities of carrier dynamics in high-frequency semiconductor devices. In this work we review several examples in which the noise performance of diodes and transistors contains information about some specific dynamics of electron and holes. In particular, we will deal with shot-noise suppression in ballistic and diffusive diodes, noise in Gunn diodes just before the onset of oscillations, plasmonic noise in FETs, kink-effect-related noise in HEMTs of different technologies and noise related to carriers injected at the gate of FETs.
\end{abstract}

Keywords-Monte Carlo simulation, Gunn oscillations, shot noise, kink effect, plasma oscillations, THz detection.

\section{INTRODUCTION}

Electronic noise is a limiting factor in many applications of semiconductor devices and integrated circuits. The fluctuations of electronic signals around their average (or cyclostationary) value can make impossible the recognition of the signal being processed or detected, that could remain hidden if the noise generated by the device or the circuit is too high. This can be particularly critical when working at high frequency with lowpower signals.

Fluctuations reflect the presence of the many processes taking place at a microscopic level and having an influence on macroscopic quantities like voltages or currents. Indeed, the level of noise and its frequency dependence may contain very useful information about such microscopic processes, in particular about carrier dynamics inside electronic devices, not available through the knowledge of the DC behavior [1].

To extract such information from the noise, it is necessary to have an exact comprehension of the microscopic processes at the origin of fluctuations and how they are transferred to the

This work has been partially supported by the Dirección General de Investigación (MICINN) through the Project TEC2013-41640-R and by the Consejería de Educación de la Junta de Castilla y León through the Project SA052U13. terminals of the devices. To this end, the use of particle simulation approaches to analyze noise in semiconductor devices [2], like the Monte Carlo (MC) method [3], can be especially useful, since they allow identifying the link between the noise behavior of the devices and the peculiarities of carrier dynamics. Indeed, within the MC technique no assumption is made about the noise sources, which are intrinsically taken into account.

In this paper we review several cases of interest. (i) The level of shot-noise suppression/enhancement in ballistic and diffusive conductors and devices contains information about injection statistics, the presence of space-charge limited transport or the energy dependence of the probability of the dominating scattering processes [4-6]. (ii) An anomalous increase of low-frequency noise can be employed as a useful indicator of current instabilities and oscillations in Gunn diodes, especially when such oscillations are not easy to detect (THz regime) [7-10]. (iii) Noise resonances can evidence the presence of collective phenomena like plasma effects in diodes and transistors, than can be exploited for signal detection [11, 12]. (iv) The location of holes generated by impact ionization in HEMTs, leading to kink effect in the output characteristics, originates a particular behavior of high-frequency noise, with a cut-off frequency related to the recombination time [13-16]. (v) Finally, electrons injected at the gate of HEMT transistors can lead to a significant noise enhancement in the drain current [17]. In all these cases, useful information on carrier dynamics can be extracted from the knowledge of the noise behavior of the devices.

\section{Monte CARlo Models AND Noise CAlculation}

The MC technique is quite appropriate not only for the analysis of the static and dynamic behaviour of high-frequency electronic devices, but also to study their noise performance. Since the description of transport is carried out at a microscopic level, it allows identifying the peculiarities of carrier dynamics and its relation to the noise behavior.

In this paper, results on different devices will be shown, going from simple two-terminal homogeneous diodes analyzed with a 1D model, through more complex self-switching diodes (SSDs) that require a $2 \mathrm{D}$ simulation, to advanced HEMT structures with an intricate geometry; every one requiring particular ingredients to be implemented in the MC models. All of them have in common the use of the simulation of an 
ensemble of charge carriers (mostly electrons, in some cases also holes) self-consistently coupled with the solution of Poisson Equation to account for the electrostatic interactions. The MC transport description is typically based on nonparabolic spherical valleys and include ionized impurity, alloy, polar and non-polar optical phonon, acoustic phonon and intervalley scattering, and appropriate carrier injection techniques at the contacts $[18,19]$. In some of the devices (SSDs and HEMTs), a negative surface charge density $\sigma$ is assigned to the semiconductor-air interfaces to account for the influence of the surface states originated during the etching processes. In the case of HEMTs, where the influence of the surface charges on the device behaviour is not critical, they are included in a simple way by fixing the value of $\sigma$ to the experimentally-extracted equilibrium value, keeping it constant independently of the topology of the structure, position along the interface, bias and time. In the case of SSDs, the surface states at the semiconductor-air interfaces are updated locally during the simulation according to the surrounding carrier concentration and operating conditions [20]. Simulations are performed at $T=300 \mathrm{~K}$.

Noise calculations are carried out in the standard way. First, from the time series of instantaneous current values obtained from the simulation, we evaluate the autocorrelation function of current fluctuations, $C_{I}(t)$. Then, by Fourier transform, the corresponding spectral density, $S_{I}(f)$, is calculated [2]. To this end, the dynamics of electrons in the device are simulated during a long enough time series divided into short time steps.

In order to model $\mathrm{THz}$ detection (AC to DC conversion), a sinusoidal signal of varying frequency is superimposed to the DC bias of a given terminal and the average current is recorded. In particular, in the case of HEMTs, as in [21], the average drain current is recorded when the sinusoidal signal is superimposed to the DC gate bias, $V_{g s}$.

\section{CASES OF INTEREST}

\section{A. Noise in Ballistic and Diffusive Conductors}

Here we analyze transport in a structure (Fig. 1) consisting in a lightly-doped active region of a semiconductor sample of length $L$ sandwiched between two heavily doped contacts (of the same semiconductor) which act as reservoirs by injecting carriers with Poissonian statistics into the active region [19]. Scattering mechanisms, considered isotropic, are introduced in the simulation in a simple way by making use of a relaxation time depending on electron energy as $\tau(\varepsilon)=\tau_{0} \varepsilon^{\alpha}$. In principle, the exponent $\alpha$ can take any value, although only some particular values correspond to well-known scattering mechanisms in a semiconductor model. As examples, $\alpha=-1 / 2$ corresponds to scattering with acoustic phonons by deformation potentials, $\alpha=0$ to neutral impurities, $\alpha=1 / 2$ to acoustic piezoelectric phonons, and $\alpha=3 / 2$ to short-range ionized impurity scattering [22].

Initially, we consider separately the case of elastic and inelastic (completely thermalizing) interactions and $\alpha=0$ (energy independent scattering time). While $L$ remains constant, the value of $\tau_{0}$ is appropriately varied covering both the ballistic and diffusive transport regimes. The transition between these regimes is described by the ratio between the

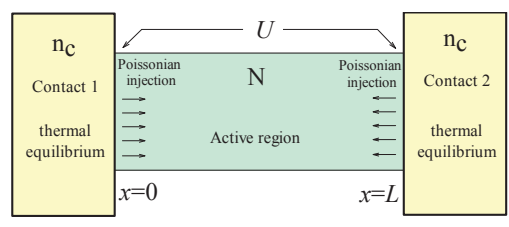

Figure 1. Schematic drawing of the structure under investigation.

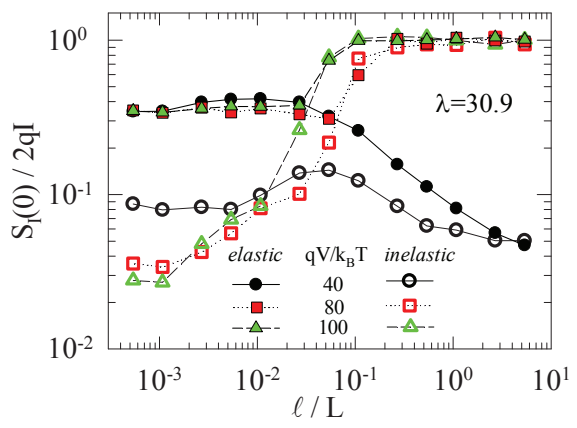

Figure 2. Fano factor vs ballistic parameter $\ell / L$ for the cases of elastic and inelastic scattering at different applied voltages. From Ref. [4].

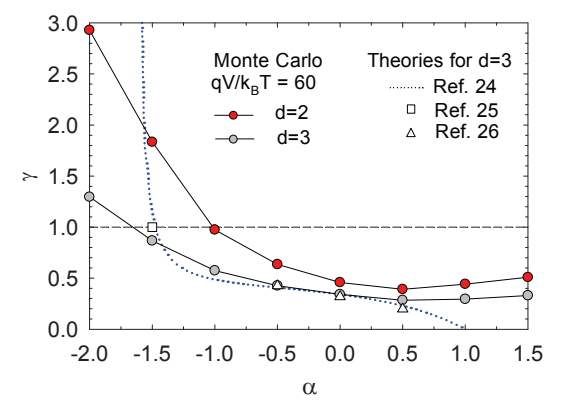

Figure 3. Fano factor as a function of the energy exponent of the elastic scattering time $\alpha$. Red and grey circles refer to MC simulations performed at $V=60 k_{B} T / q$ for $d=3$ and $d=2$, respectively. Dotted curve and other symbols refer to analytical theories carried out under spacecharge limited conditions. From Ref. [5].

carrier mean free path $\ell$ and the sample length $L$. The level of space charge inside the active region is characterized by the dimensionless parameter $\lambda$, defined as $L / L_{D c}$, where $L_{D c}$ is the Debye length corresponding to the carrier concentration at the contacts [23]. A high value of $\lambda$ indicates space-charge limited transport.

Fig. 2 reports the Fano factor $\gamma=S_{I}(0) / 2 q I$ as a function of $\ell / L$ for several values of the applied voltage $V$ in the case of elastic and inelastic scattering. In the perfect ballistic regime the two distinct values of $\gamma$ inform about the presence or absence of the potential barrier limiting the current due to space charge [23]. For $V=40 k_{B} T / q$ the barrier is still present and the suppression is important. For $V=80 k_{B} T / q$ and 100 $k_{B} T / q$ the barrier has already disappeared; accordingly the current saturates and the suppression factor takes the full shotnoise value. When the diffusive regime is achieved, in the elastic case $\gamma$ attains a constant value at further decreasing of $\ell / L$, and takes the same value of about $1 / 3$ independently of the applied voltage. On the contrary, in the inelastic case the higher the applied voltage the lower the value finally reached by $\gamma$. 
The limiting value reached by $\gamma$ in the elastic diffusive limit (approximately $1 / 3$ in the case of $\alpha=0$ ) provides also useful information, in this case about the energy dependence of the dominating scattering mechanism, as shown in Fig. 3, where $\gamma$ is plotted as a function of $\alpha$ in such a limit. Results for two different dimensionalities of momentum space $d=2,3$ are provided. The figure also includes different theoretical predictions developed to interpret MC results [24-26]. As observed, a significant dependence of $\gamma$ on both $\alpha$ and $d$ exists, reflecting the signature of scattering mechanisms on the noise of the structure. The Fano factor is found to exhibit an asymmetric behavior with $\alpha$, showing a tendency to saturate at positive values of $\alpha$ (taking suppressed values near to $1 / d$ ), while increasing systematically towards enhanced values well above unity (noise enhancement) at the largest negative values of $\alpha$.

\section{B. Noise as Precursor of Gunn Oscillations}

A Gunn diode oscillates when the bias voltage exceeds a threshold value. However, between the initial static state and the generation of a purely periodic signal there exists a voltage range for which incoherent fluctuations appear, leading to an significant increase of noise at frequencies much lower than those of the oscillations. This effect has been predicted by MC simulations [7-9] and recently confirmed experimentally [10]. The origin of the noise enhancement is attributed to the existence of intermittent oscillations for a given range of voltages, preceding the formation of a coherent oscillation. In practice, such a noise increase can be used as an indirect method to predict higher-frequency oscillations in setups where a direct detection is a complicated challenge, as in the case of (sub-)THz Gunn oscillators. That is the case illustrated here, where simulations of GaN asymmetric diodes, so called SSDs [27], with the geometry shown in Fig. 4, are performed. Details about the model can be found in [9]. The high saturation velocity of $\mathrm{GaN}$ allows oscillation frequencies approaching the $\mathrm{THz}$ range for channel lengths about a micron.

Fig. 5 shows (a) $I-V$ and (b) $S_{I}(0)-V$ curves for three SSDs with different values of the surface charge density $\sigma$. This parameter is decisive for achieving current oscillations that present harmonics from $100 \mathrm{GHz}$ up to $1 \mathrm{THz}$. Current sequences and noise spectra are also plotted as insets in order to get more information about what happens in the diode for several voltages at which $S_{I}(0)$ exhibits significant variations. A higher value of $\sigma$ leads to a stronger channel depletion, which makes necessary higher voltages to achieve, first, current conduction through the diode and, then, Gunn oscillations, as shown in Fig. 5(a). We note that at such voltages there are no kinks in the I-V curves. Let us focus on the diode with lower value of surface charge density, SSD1. As can be observed, the threshold for the onset of Gunn oscillations is about $40 \mathrm{~V}$. Below this voltage, starting from the Nyquist value at equilibrium, the noise exhibits a slight increase with the bias due to electron heating. For voltages higher than $36 \mathrm{~V}$, a remarkable enhancement of the low-frequency noise is detected, in parallel to the transition from a passive- to a generation-state as evidenced by the current sequence and its corresponding spectral density at $40 \mathrm{~V}$. Once the oscillations are well established (voltages above $45 \mathrm{~V}), S_{I}(0)$ decreases, and

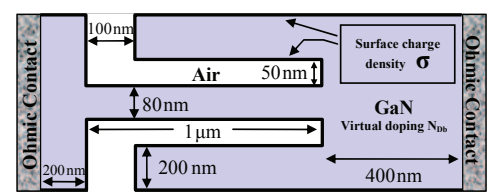

Figure 4. Scheme of the top-view simulations of the channel with the involved parameters, background doping $\mathrm{N}_{\mathrm{Db}}$ and surface charge $\sigma$, and the considered dimensions of the diode.

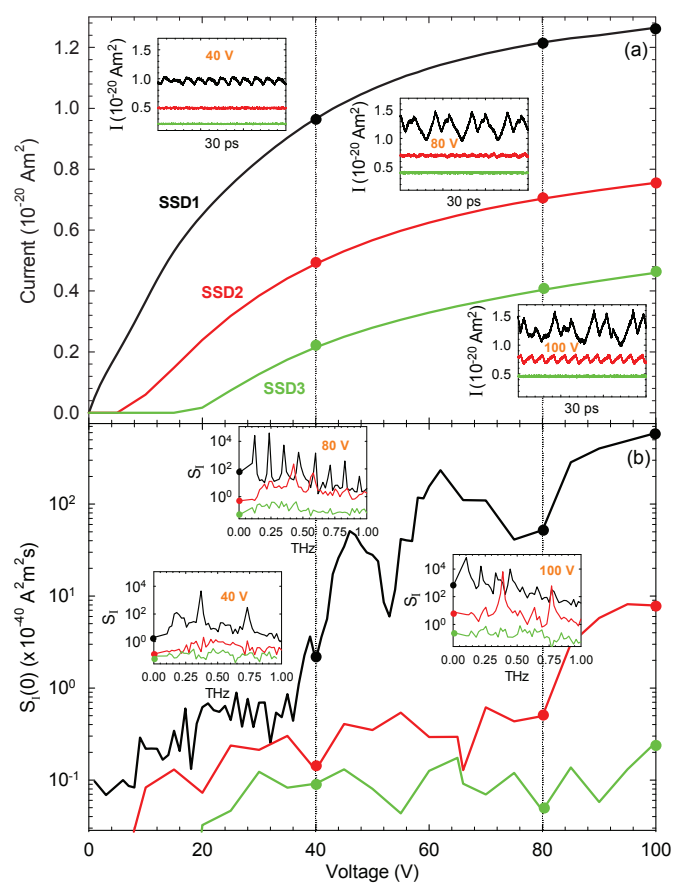

Figure 5. (a) I-V curves and (b) low-frequency value of the current spectral density for SSD1, that has $\sigma / \mathrm{q}=-0.25 \times 10^{12} \mathrm{~cm}^{-2}$, and two more diodes, SSD2 and SSD3, with $\sigma / \mathrm{q}=-1.0 \times 10^{12} \mathrm{~cm}^{-2}$ and $\sigma / \mathrm{q}=-1.5 \times 10^{12}$ $\mathrm{cm}^{-2}$, respectively. The insets in (a) show current sequences for some applied voltages and in (b) the corresponding spectra. From Ref. [9].

then, at around $53 \mathrm{~V}$, it increases again because of the onset of further frequency components. For higher voltages we find once more the previously explained behaviour, i.e., a drop in the noise up to $75 \mathrm{~V}$ due to the presence of clear oscillations followed by another rise according to the incorporation of more harmonics, as occurs at $100 \mathrm{~V}$.

Simulations of SSD2 and SSD3, with increasing values of surface charge, confirm that the enhancement of the lowfrequency noise is certainly due to the onset of current oscillations and further spectral components. In SSD2 oscillations are more difficult to be obtained than in SSD1, being necessary the application of a voltage around $80 \mathrm{~V}$, while for SSD3 the effective width of the channel is not enough to achieve current oscillations even at $100 \mathrm{~V}$. In fact, the current level is much lower than in the other diodes. With regard to the low-frequency noise [see Fig. 5(b)], the curve corresponding to SSD2 exhibits a more or less constant value up to $80 \mathrm{~V}$ (with a slight increase due to electron heating) and then rises considerably, coinciding with the onset of oscillations at that 
voltage. For a lower value of $\sigma$, which is the case of SSD1 discussed before, such a bump takes place at lower bias, $36 \mathrm{~V}$, while for a higher value of $\sigma$, as occurs for $\operatorname{SSD} 3, S_{I}(0)$ remains practically constant since no oscillation is achieved.

\section{Noise and THz Rectification in HEMTs}

Plasma-related charge fluctuations in regions where a potential barrier limits the current flowing through the devices originates a link between the noise in the current (given by the plasma oscillations) and the $\mathrm{AC}$ to $\mathrm{DC}$ rectification (given by the coupling between the internal plasma and the external output signal). This effect takes place in diodes, like SSDs [11], and transistors [12], and allows identifying resonances in the noise that could be exploited for signal detection. This approach has been confirmed by experiments made with classic HEMTs based on different material systems (GaAs, InGaAs and $\mathrm{GaN}$ ) and Si MOSFETs, where $\mathrm{THz}$ detection as a result of plasma wave resonances has been demonstrated [28].

Here we show some illustrative results in HEMTs based on InGaAs channels, with the geometry shown in Fig. 6. The comparison between simulations of noise and $\mathrm{THz}$ detection allows determining the location of the dominant plasma effects leading to signal detection. Additionally, information about the plasma modes (2D and $3 \mathrm{D}$, gated and ungated) present in the structure can be obtained [12].

Fig. 7 compares the spectral densities of (a) source, (b) gate, and (c) drain current fluctuations with the frequency dependent DC drain current resulting from the superposition of a sinusoidal signal at the gate terminal in HEMTs with different length of the source access region $L_{S}$. As observed, the noise spectra exhibit a resonance related to plasma effects, which seems to be also present in the rectification of the drain current. The dependence on $L_{\mathrm{S}}$ of the amplitude and frequency of the peak in the AC to DC conversion signal is clearly correlated with that of the noise spectra of the source current [Fig. 7(a)], but not with the gate and drain ones. This happens because it is the resonance between the plasma-related charge fluctuations of the source-gate region and the oscillating gate voltage that results in the increase of the average drain current, $\Delta I_{\mathrm{d}}$, due to an enhanced injection of electrons over the potential barrier induced by the gate electrode. In fact, the very same characteristic frequency is also visible (not shown here) in the spectra of the electric potential fluctuations in the channel under the source side of the gate (the point that determines the value of $I_{\mathrm{d}}$ since it is where the carrier injection over the potential barrier takes place). Moreover, both the gate-drain distance and the gate length do not affect the position of the resonant peak of the AC to DC conversion. On the other hand, in addition to the previously observed dependence on $L_{\mathrm{S}}$, the resonant frequency strongly depends on the length of the source side of the recess, $L_{\mathrm{r}}$. These results clearly indicate that the source-gate region (including the recess) acts as the plasma wave cavity that produces the resonant detection of $\mathrm{THz}$ radiation in HEMTs, fact that is also reflected in the source current noise spectra.

\section{Kink-effect Related Noise in HEMTs}

The kink effect consists in an anomalous increase in the drain current $I_{\mathrm{d}}$ at sufficiently high drain-to-source voltages $V_{d s}$, which leads to a reduction in the gain and a rise in the level of

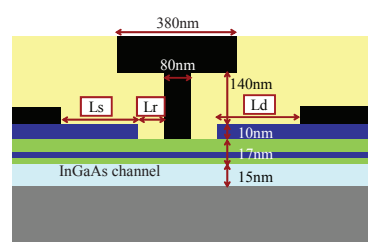

Figure 6. Layer structure and geometry of the InGaAs HEMTs.
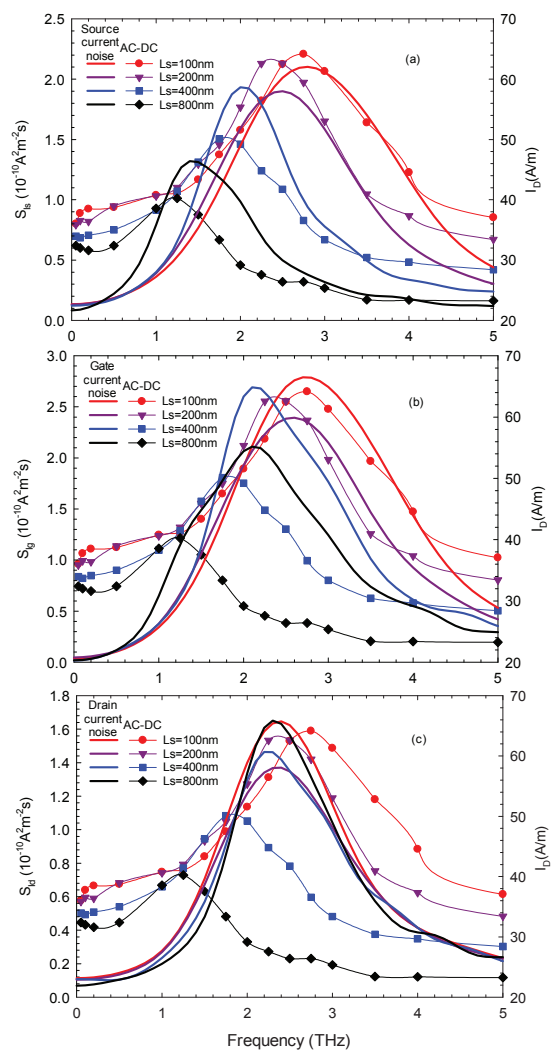

Figure 7. Comparison between the (a) source, (b) gate and (c) drain current noise spectra, $S_{I s}, S_{I g}$ and $S_{I d}$, respectively, and the frequency dependent drain current due to AC to DC conversion, calculated as the average drain current originated by the superposition of a sinusoidal signal of $0.1 \mathrm{~V}$ of amplitude and varying frequency to the $\mathrm{DC}$ gate potential $\left(V_{\mathrm{gs}}=-0.3 \mathrm{~V}, V_{\mathrm{ds}}=1.0 \mathrm{~V}\right)$ of a HEMT with $L_{\mathrm{r}}=100 \mathrm{~nm}$ and $L_{\mathrm{d}}=500 \mathrm{~nm}$ and different values of $L_{\mathrm{s}}$.

noise [13]. Here we will describe the behavior of the noise related to the kink effect in two HEMTs fabricated with different technologies: a $100 \mathrm{~nm}$-gate InGaAs/InAlAs HEMT $[13,29]$ and a 225-nm isolated-gate InAs/AlSb HEMT [15, 30]. Even if the influence of kink effect on the DC, AC and noise behavior of the transistors is similar for both technologies, its physical origin exhibits significant differences, mainly related to the location inside the device of the holes (generated by impact ionization) at the origin the effect. For the analysis of this phenomenon is therefore essential to include in the MC model impact ionization events as well as hole recombination [13]. Good agreement between the MC simulations and experimental measurements of output characteristics, not shown here, where obtained for both transistors $[13,15]$. 

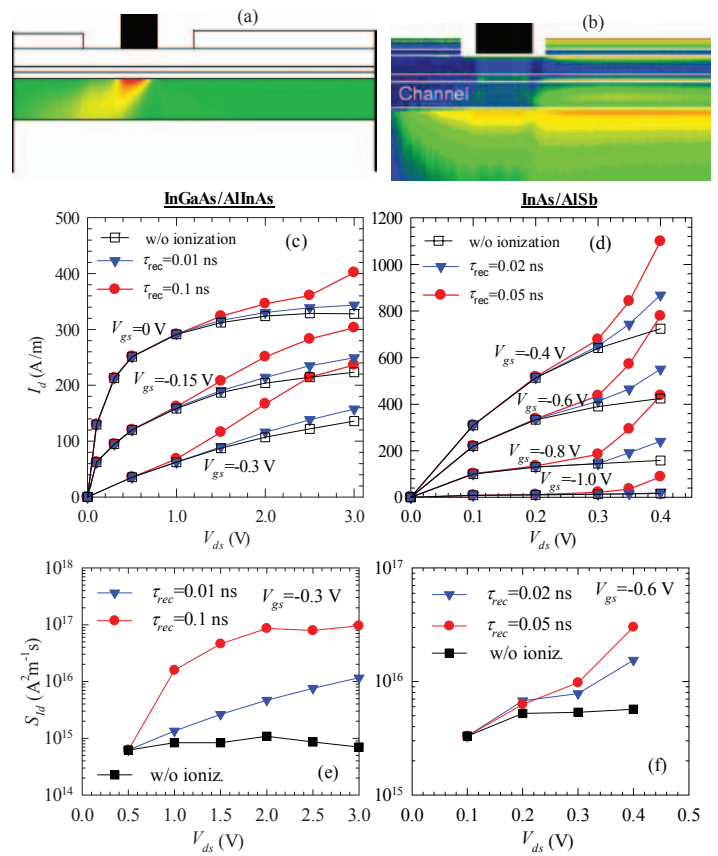

Figure 8. Hole density map for the (a) InGaAs and (b) InAs HEMTs. (c) and (d) output characteristics and (e) and (f) $S_{I d}(0)$ vs. $V_{\text {ds }}$ obtained from $\mathrm{MC}$ simulations with and without considering impact ionization mechanisms. (c) and (e) correspond to the InGaAs HEMT, (d) and (f) to the InAs one. Two different values for the recombination time $\tau_{\text {rec }}$ have been used: 0.01 and $0.1 \mathrm{~ns}$ for the InGaAs HEMT, and 0.02 and $0.05 \mathrm{~ns}$ for the InAs-HEMT.

MC simulations allow identifying the physical origin of the kink effect as follows. For high enough $V_{d s}$ the holes generated by impact ionization mechanisms, mainly in the gate-drain region, move towards the source contact. This happens for $V_{d s}$ about $0.7 \mathrm{~V}$ and $0.3 \mathrm{~V}$ for InGaAs and InAs HEMTs, respectively, when the gate-to-drain voltage provides the electrons with a kinetic energy similar to the bandgap of the channel semiconductor $(0.75 \mathrm{eV}$ for InGaAs and 0.35 for InAs). In the case of InGaAs HEMTs, the generated holes are confined in the channel (type I heterojunction) and tend to pile up under the source side of the gate, Fig. 8(a). For the InAsHEMT, the valence band discontinuity and the electric field benefit the descent of the holes towards the AlSb buffer (type II heterojunction), where they accumulate, mainly under the gate and at the gate-drain region of the transistor, Fig. 8(b). Due to this pile-up of positive charge, the potential barrier which controls the passage of electrons through the channel is lowered, thus the channel is further opened and $I_{d}$ increases, leading to the kink effect in the output characteristics.

In InAs HEMTs, the increase of $I_{d}$ for a fixed $V_{d s}$ due to the appearance of holes grows with $V_{g s}$. This behavior is the opposite to that found for InGaAs devices, in which the increase of $I_{d}$ is lower for higher $V_{g s}$, which occurs because although the electron concentration in the channel is larger when the channel opens, the maximum electron energy is reduced due to the lower gate-to-drain potential. In the case of InAs HEMTs the bandgap is much smaller, thus impact ionization probability remains significant for higher values of

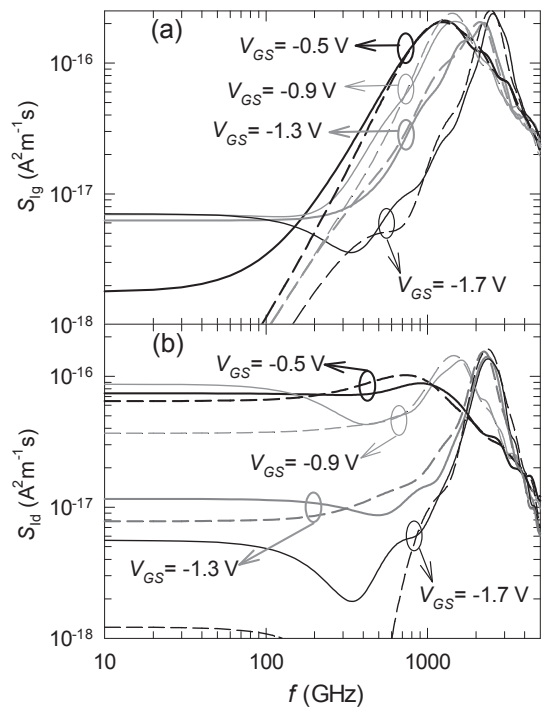

Figure. 9. (a) $S_{I g}(f)$ and (b) $S_{I d}(f)$ with (solid lines) and without (dashed lines) considering gate-tunnel injection. $V_{d s}=1.0 \mathrm{~V}$. From Ref. [17].

$V_{g s}$. Besides, impact ionization events take place not only near the maximum of electron energy (under the gate), but all along the drain-side of the channel, where the electron velocity is higher when increasing $V_{g s}$

Apart from this static effect, impact ionization and hole recombination lead to fluctuations of the hole concentration in the channel, particularly in the pile-up. Since these charge fluctuations are strongly coupled to the drain-current fluctuations by the high transconductance of the transistor, an important increase of the drain-current noise is expected to take place concurrently with the kink in the $I_{d}-V_{d s}$ curves. Thus, the low-frequency value of $S_{I d}$, Figure $8(\mathrm{e})$ and (f), is found to increase with $V_{d s}$ (due to the higher number of impact ionization events) and $\tau_{\text {rec }}$, both enhancing the pile-up of holes, with a strategic position under the gate, Figure 8(a) and (b). Indeed, $S_{I d}(0)$ depends on $V_{d s}$ and $V_{g s}$ in a similar way to that shown by the kink-related increase of $I_{d}$ : more pronounced for higher $V_{d s}$ and lower (higher) $V_{g s}$ in InGaAs (InAs) HEMTs. However, the relative increase of $S_{I d}(0)$ with respect to its value when impact ionization is not considered in the simulations is much higher than that of $I_{d}$. And even in conditions where the kink effect in the $I_{d}-V_{d s}$ curves is hardly detectable [for example when $V_{d s}$ is just above the onset of impact ionization mechanisms, $V_{d s}=1.0 \mathrm{~V}$ for the InGaAs HEMT, Fig. 8(c), and $V_{d s}=0.3 \mathrm{~V}$ for the InAs HEMT, Figure 8(d)], $S_{I d}(0)$ already exhibits a significant increase [Fig. 8(e) and (f)]. This indicates that when HEMTs are biased in the vicinity of the kink onset, even if the static behavior of the transistors is not perturbed, their noise is already showing the signature of the effect, since drain noise is extremely sensitive to the dynamics of holes generated by impact ionization.

\section{E. Noise Related to Gate Electron Injection in HEMTs}

Finally, here we explain how the tunnel injection of electrons through the gate in a $100 \mathrm{~nm}$-gate InGaAs/InAlAs HEMT modifies the noise spectra [17]. Fig. 9 shows (a) $S_{I g}$ 
and (b) $S_{I d}$ as a function of frequency, with and without considering gate tunnel injection in the device, for several biasing conditions. The high-frequency peak due to plasma oscillations is noticed in all cases. As observed, $S_{I d}$ presents an increase of the low-frequency plateau when tunnel effect is included in the simulations, more pronounced the lower (more negative) is $V_{g s}$ and thus more intensive electron injection takes place. Interestingly, there is an increase in $S_{I d}$ due to the gate tunnel injection while this effect leads to a reduction in $I_{d}$ in open channel conditions. Again, like at the onset of kink effect, this increase in $S_{I d}$ is remarkable even when the tunnel effect is hardly noticeable in the $I-V$ characteristics (not shown). The origin of this effect in the noise is attributed to the fluctuations induced in the potential barrier controlling the current flow through the channel by the electrons injected under the gate into the Schottky layer. These potential fluctuations are strongly coupled with the drain current fluctuations, leading to the large increase observed in $S_{I d}$. As concerns $S_{I g}$, due to the appearance of a DC $I_{g}$ component originated by the tunnel injection through the Schottky contact, a low-frequency enhancement in $S_{I g}$, roughly corresponding to the full shot noise value $2 \mathrm{q} I_{g}$, takes place.

\section{ACKNOWLEDGMENTS}

The author gratefully acknowledges helpful discussions and critical reading of the manuscript by $J$. Mateos and the contribution to the reported results of present and former members of the Semiconductor Devices Research Group at Salamanca University (http://www.usal.es/gelec) and rest of collaborators belonging to other international groups.

\section{REFERENCES}

[1] R. Landauer, "The noise is the signal," Nature, vol. 392, pp. 658-659, 1998.

[2] L. Varani, L. Reggiani, T. Kuhn, T. González, and D. Pardo, "Microscopic simulation of electronic noise in semiconductor materials and devices," IEEE Trans. Electron Dev., vol. 41, pp.1916-1925, 1994.

[3] C. Jacoboni and P. Lugli, The Monte Carlo Method for Semiconductor Device Simulation, Vienna, Springer-Verlag, 1989.

[4] T. González, J. Mateos, D. Pardo, O. M. Bulashenko, and L. Reggiani, "Microscopic analysis of shot-noise suppression in nondegenerate diffusive conductors," Phys. Rev. B, vol. 60, pp. 2670-2679, 1999.

[5] G. Gomila, T. González, and L. Reggiani, "Shot-noise anomalies in nondegenerate elastic diffusive conductors," Phys. Rev. B, vol 66, pp. $2454231-8,2002$.

[6] G. Iannaccone, A. Betti, and G. Fiori, "Suppressed and enhanced shot noise in one dimensional field-effect transistors," J. Comput. Electron., vol.14, pp 94-106, 2015.

[7] E. Starikov, P. Shiktorov, V. Gruzinskis, L. Reggiani, L.Varani, and J. C. Vaissiere, "Noise Enhancement as Indicator of Instability Onset in Semiconductor Structures," AIP. Conf. Proc., vol. 780, pp. 791-794, 2005.

[8] P. Shiktorov, E. Starikov, V. Gruzinskis, L. Varani, and L. Reggiani, "Giant enhancement of low-frequency noise as precursor for the onset of a high-frequency instability," AIP Conf. Proc., vol. 1129, pp. 179-182, 2009.

[9] A. Íñiguez-de-la-Torre, I. Íñiguez-de-la-Torre, J. Mateos, and T. González, "Correlation between low-frequency current-noise enhancement and high-frequency oscillations in GaN-based planar nanodiodes: A Monte Carlo study," Appl. Phys. Lett., vol. 99, pp. $0621091-3,2011$.

[10] O. García-Pérez, Y. Alimi, A. M. Song, I. Iñiguez-de-la-Torre, S. Pérez, J. Mateos, and T. González, "Experimental assessment of anomalous low-frequency noise increase at the onset of Gunn oscillations in
InGaAs planar diodes," Appl. Phys. Lett., vol. 105, pp. 113502 1-4, 2014.

[11] I. Iñiguez-de-la-Torre, J. Mateos, D. Pardo, A. M. Song, and T. González, "Noise and terahertz rectification linked by geometry in planar asymmetric nanodiodes," App. Phys. Lett., vol. 94, 093512 1-3, 2009.

[12] J. Mateos and T. González, "Plasma enhanced terahertz rectification and noise in InGaAs HEMTs," IEEE Trans. THz Sci. Technol., vol. 2, pp. 562-569, 2012.

[13] B. G. Vasallo, J. Mateos, D. Pardo, and T. González, "Monte Carlo study of the kink effect in short-channel InAlAs/InGaAs high electron mobility transistors," J. Appl. Phys., vol. 94, pp. 4096-4101, 2003.

[14] B. G. Vasallo, J. Mateos, D. Pardo, and T. González, "Kink-effect related noise in short-channel InAlAs/InGaAs high electron mobility transistors," J. Appl. Phys., vol. 95, pp. 8271-8274, 2004.

[15] B. G. Vasallo, H. Rodilla, T. González, G. Moschetti, J. Grahn, and J. Mateos, "Monte Carlo study of kink effect in isolated-gate InAs/AlSb high electron mobility transistors," J. Appl. Phys., vol. 108, pp. 094505 $1-5,2010$.

[16] B. G. Vasallo, H. Rodilla, T. González, G. Moschetti, J. Grahn, and J. Mateos, "Kink effect and noise performance in isolated-gate InAs/AlSb High Electron Mobility Transistors," Semicond. Sci. Technol., vol. 27, pp. 065018 1-5, 2012.

[17] D. Moro-Melgar, J. Mateos, T. González, and B. G. Vasallo, "Effect of tunnel injection through the Schottky gate on the static and noise behavior of GaInAs/AlInAs HEMTs," J. Appl. Phys., vol. 116, pp. $2345021-7,2014$.

[18] T. González and D. Pardo, "Physical models of ohmic contact for Monte Carlo device simulation," Solid-State Electron., vol. 39, p. 555, 1996.

[19] T. González, J. Mateos, D. Pardo, L. Varani, and L. Reggiani, "Injection statistics simulator for dynamic analysis of noise in mesoscopic devices," Semicond. Sci. Technol., vol. 14, pp. L37-L40, 1999.

[20] I. Iñiguez-de-la-Torre, J. Mateos, T. González, D. Pardo, S. Bollaert, Y. Roelens, and A. Cappy, "Influence of the surface charge on the operation of ballistic T-branch junctions: a self-consistent model for Monte Carlo simulations," Semicond. Sci. Technol., vol. 22, pp. 663670, 2007.

[21] M. Dyakonov and M. S. Shur, "Detection, mixing, and frequency multiplication of Terahertz radiation by two dimensional electronic fluid," IEEE Trans. Electron Dev., vol. 43, pp. 380-387, 1996.

[22] K. Seeger, Semiconductor Physics, an Introduction, Berlin: Springer, 1985.

[23] T. González, J. Mateos, D. Pardo, O. M. Bulashenko, and L. Reggiani, "Microscopic analysis of shot-noise suppression in nondegenerate ballistic transport," Semicond. Sci. Technol., vol. 13, pp. 714-724, 1998.

[24] C. W. J. Beenakker, "Sub-Poissonian shot noise in nondegenerate diffusive conductors," Phys. Rev. Lett., vol. 82, pp. 2761-2763, 1999.

[25] K. E. Nagaev, "Comment on "Universality of the $1 / 3$ shot-noise suppression factor in nondegenerate diffusive conductors," Phys. Rev. Lett., vol. 83, p. 1267, 1999.

[26] V. L. Gurevich and M. I. Muradov, "The theory of shot noise in the space-charge-limited diffusive conduction regime," J. Exp. Theor. Phys., vol. 94, pp. 1026-1034, 2002.

[27] A. M. Song, M. Missous, P. Omling, A. R. Peaker, L. Samuelson, and W. Seifert, "Unidirectional electron flow in a narrow semiconductor channel: A self-switching device," Appl. Phys. Lett., vol. 83, pp. 1881$1883,2003$.

[28] W. Knap, M. Dyakonov, D. Coquillat, F. Teppe, N. Dyakonova, J. Łusakowski, K. Karpierz, M. Sakowicz, G. Valusis, D. Seliuta, I. Kasalynas, A. El Fatimy, Y. M. Meziani, and T. Otsuji, "Field Effect Transistors for Terahertz Detection: Physics and First Imaging Application,” J. Infrared Milli. Terahz Waves, vol. 30, pp. 1319-1337, 2009.

[29] J. Mateos, D. Pardo, T. González, V. Hoel, and A. Cappy, "Monte Carlo Simulator for the Design Optimization of Low-Noise HEMTs," IEEE Trans. Electron. Dev., vol. 47, pp. 1950-1956, 2000.

[30] M. Malmkvist, S. Wang, and J. Grahn "Epitaxial optimization of 130$\mathrm{nm}$ gate-length InGaAs/InAlAs/InP HEMTs for high-frequency applications," IEEE Trans. Electron Dev., vol. 55, pp.268 -275, 2008. 